\title{
INOVAÇÃO NA DISPONIBILIDADE DOS SISTEMAS DE NÍVEL 2 DE OTIMIZAÇÃO DE PROCESSO GARANTINDO A ESTABILIDADE OPERACIONAL DAS UNIDADES FABRIS*
}

Leandro Rodrigues Ramos ${ }^{1}$

Alexander de Oliveira Botelho ${ }^{2}$

\section{Resumo}

Os Sistemas de Nível 2 de Otimização da ArcelorMittal Tubarão impactam diretamente no processo produtivo (Qualidade, Produtividade, Produção), sendo que parte considerável destes sistemas são executados em plataforma legada de hardware (Alpha Digital). Em 2011/2012, o fim do ciclo de vida da Plataforma Alpha (Open VMS) anunciado pela HP trouxe este tópico para um lugar de destaque na matriz de risco da Engenharia de Automação. A principal estratégia é a migração para novas plataformas, porém associa-se a esta opção um custo e tempo elevado, principalmente se tratando de sistemas de alta complexidade e disponibilidade. A gestão de um estoque de sobressalentes também se torna cada vez mais difícil devido à escassez destes equipamentos no mercado global. Considerando estes fatores, a busca de soluções alternativas de curto prazo se fez necessária. Uma tecnologia de emulação e virtualização de plataforma foi validada e um projeto piloto implantado no sistema responsável pela otimização do processo de reaquecimento de placas do Laminador de Tiras a Quente. O uso desta tecnologia promoveu uma portabilidade direta do sistema para um hardware padrão de mercado (Intel x64), em curto prazo e sem impactos operacionais, podendo ser disseminada para outros sistemas, garantindo um pulmão para futuras migrações.

Palavras-chave: Alpha; Emulação; Nível 2; LTQ.

\section{INNOVATION IN AVAILABILITY OF L2 OPTIMIZATION SYSTEMS TO GUARANTEE THE OPERATIONAL STABILITY OF THE INDUSTRIAL PLANT Abstract}

The L2 Optimization systems from ArcelorMittal Tubarao directly impact in the production process, and most of them are running on a legacy hardware platform (Alpha Digital). In 2011/2012, the end of life cycle Platform Alpha (Open VMS) has been announced by HP, bringing this topic to a prominent place in the risk matrix of Automation Engineering. The main strategy is converting the systems to new platforms, but we have high costs and big timeframes associated with this option, especially when dealing with highly complex systems. The management of spare parts becomes difficult due the lack of these devices in the global market. Considering these factors, the research for short-term solutions was needed. A technology involving emulation and virtualization has been validated and a project pilot deployed in the system responsible for the optimization of slab reheating furnaces of ArcelorMittal Tubarão HSM plant. This technology promoted a direct portability of the system to a supported hardware (Intel x64), in a short time and without operational impacts, and may be disseminated to other systems, giving more time for future migrations.

Keywords: Alpha; Emulation; Level 2; HSM

1 Bacharel em Ciência da Computação. Especialista Desenvolvimento em Automação e Instrumentação. ArcelorMittal Tubarão. Tubarão, ES, Brasil.

2 Engenheiro. Especialista de Desenvolvimento de Sistemas de Automação. ArcelorMittal Tubarão. Serra, ES, Brasil

* Contribuição técnica ao $18^{\circ}$ Seminário de Automação e TI Industrial, 23 a 26 de setembro de 2014, São Paulo, SP, Brasil. 


\section{INTRODUÇÃO}

A Engenharia de Automação da ArcelorMittal Tubarão possui hoje diversos sistemas de otimização e apoio ao processo produtivo. São aplicações complexas, desenvolvidas e mantidas ao longo de vários anos, sendo que uma parte considerável destes sistemas é executada em plataforma de hardware legada (Alpha Digital). Esta obsolescência do hardware traz uma série de implicações que devem ser consideradas:

- Componentes se desgastam ao longo do tempo: Rotação de discos, aquecimento de processadores, etc;

- Crescimento no número de incidentes relacionados ao hardware;

- Aumento nos custos de manutenção;

- Inventário limitado de spare parts;

- Dificuldade em se adquirir equipamentos no mercado (Usualmente servidores recondicionados).

Como estratégia principal optou-se pela recodificação e migração destes sistemas para plataformas modernas (Java, Windows), com o ciclo de vida ativo (Figura 1). Porém migrações para uma nova plataforma impactam em custo e tempo elevados, com um grau considerável de esforço e risco.
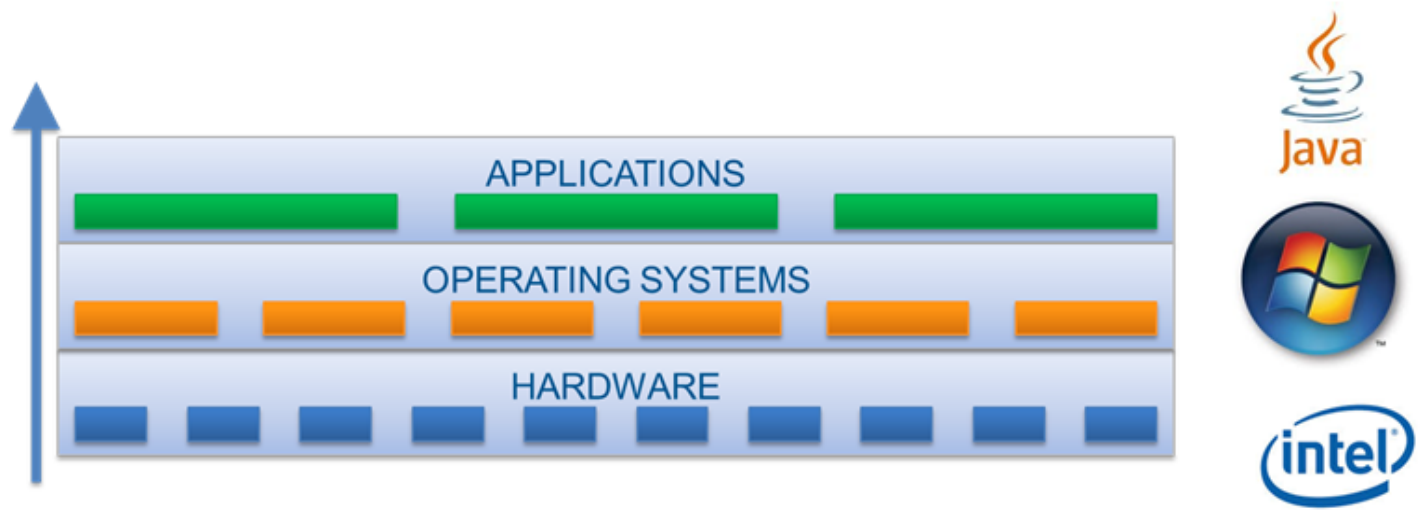

Figura 1 - Novas tecnologias propostas para os sistemas de Automação

A gestão de um estoque de sobressalentes (servidores e peças) se torna cada mais difícil devido à escassez deste tipo de equipamento no mercado global. Sendo assim, este contexto nos trouxe a necessidade de pensar em alternativas de curto prazo visando atacar o problema de se ter tecnologia legada executando aplicações de missão crítica.

Em 2012 iniciou-se uma busca no mercado por soluções alternativas, onde produtos de emulação e virtualização da plataforma foram encontrados. Estas soluções estão baseadas na transcrição das instruções do processador legado (Alpha) para uma arquitetura alvo, neste caso Intel $\times 64$.

Foram avaliados softwares desta natureza sendo que o mais aderente aos requisitos de compatibilidade/portabilidade, confiabilidade e suporte do fabricante foi ofertado pela STROMASYS Virtualization Technologies [1], denominado CHARON. Esta empresa, com sede na suíça, foi fundada por ex-funcionários da Digital Equipment Corporation (DEC), adquirida pela Hewelett Packard (HP) e detentora original da arquitetura Alpha. Ou seja, trata-se de uma empresa que possui engenheiros com profundo conhecimento sobre esta plataforma. Além disto, a STROMASYS possui uma longa parceira com própria Hewlett Packard, fornecedora atual do sistema operacional e hardware dos sistemas de otimização.

* Contribuição técnica ao $18^{\circ}$ Seminário de Automação e TI Industrial, 23 a 26 de setembro de 2014, São Paulo, SP, Brasil. 
A Figura 2 ilustra uma visão geral desta arquitetura proposta.
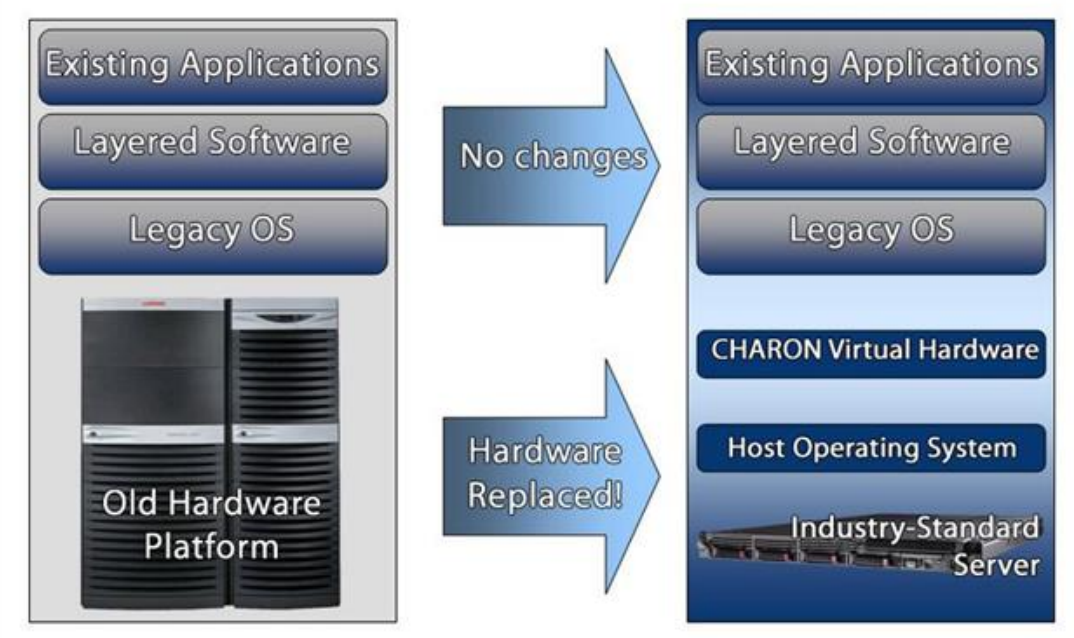

mmare PARTNER

TECHNOLOGY ALLIANCE

\section{STROMASYS}

Figura 2 - Arquitetura de Emulação utilizando o CHARON

Esta abordagem traz, em curto prazo, os seguintes benefícios:

- Emulação do hardware, promovendo portabilidade direta das aplicações e sistemas operacionais legados (Open VMS e Tru64);

- Baixo risco e esforço nas migrações;

- Transparência para os usuários finais com a retenção do conhecimento e sem impactos operacionais;

- Eliminação falhas/riscos de hardware obsoleto;

- Transição para plataforma de hardware padrão de mercado (Intel x64);

- Redução de espaço e consumo de energia (Green IT);

- Agilidade no provisionamento de ambientes de testes/desenvolvimento;

- Aumento de desempenho com a utilização de CPU mais modernas, storages e rede;

- "Pulmão" - Liberação de hardware Alpha, aumentando o estoque de sobressalentes o que nos tranquiliza para seguir com a estratégia de recodificação dos sistemas para as plataformas atuais.

A área definida para um projeto piloto com o intuito de se testar esta tecnologia foi o LTQ da ArcelorMittal Tubarão implantado em 31/08/2002. A Figura 3 mostra a visão dos principais equipamentos que compõem o laminador de tiras a quente da ArcelorMittal Tubarão, destacando-se a localização dos fornos de reaquecimento de placas.

\footnotetext{
* Contribuição técnica ao $18^{\circ}$ Seminário de Automação e TI Industrial, 23 a 26 de setembro de 2014,
} São Paulo, SP, Brasil. 

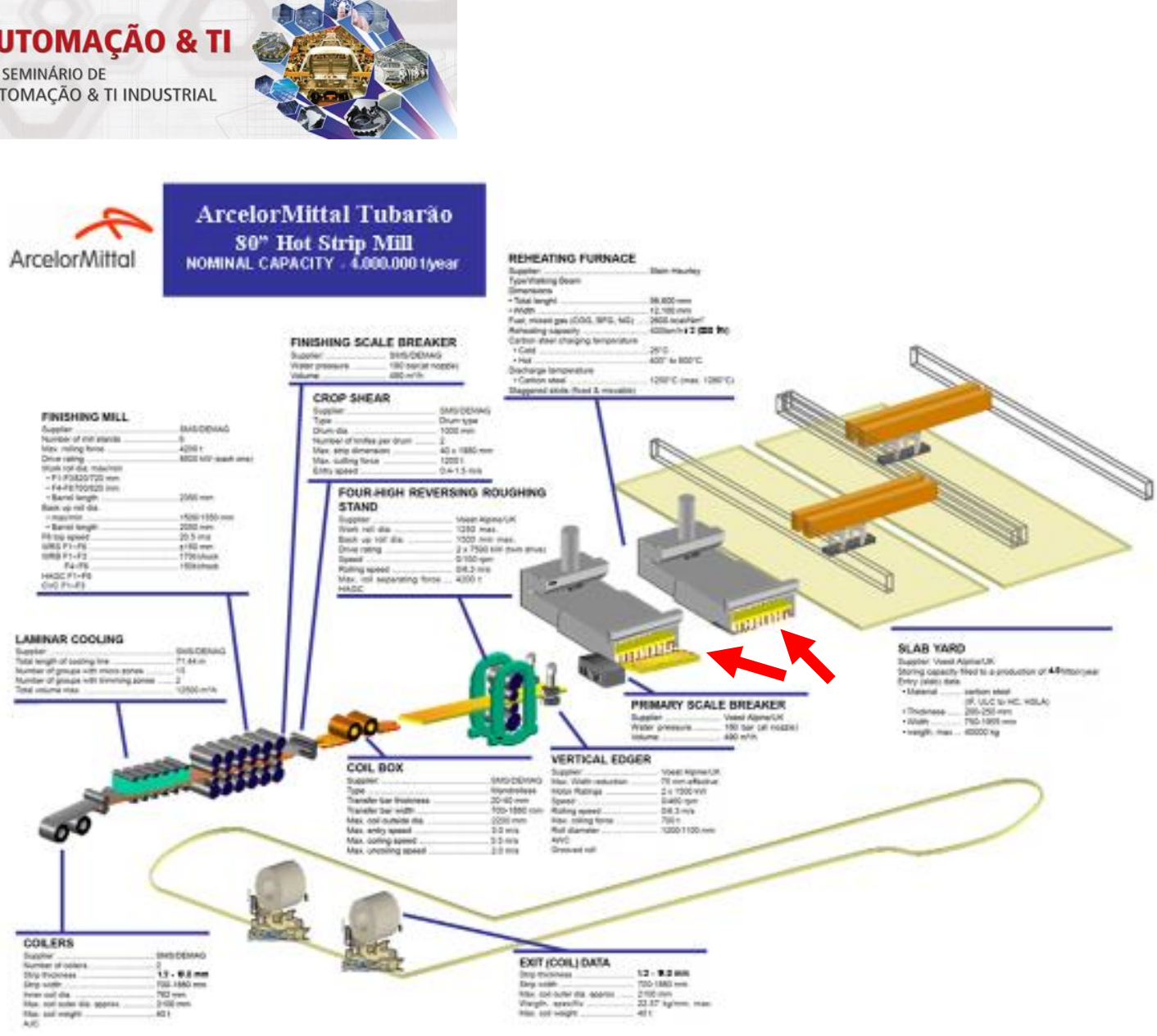

Figura 3 - Layout do LTQ da ArcelorMittal Tubarão

O Sistema de Nível 2 de otimização dos Fornos de Reaquecimento de Placas foi definido para o projeto piloto devido às sua arquitetura que permite uma comparação "on line" do ambiente de produção na plataforma legada Alpha com o ambiente de desenvolvimento em sua nova plataforma Intel x64 operando de forma paralela e simultânea, permitindo assim a validação não somente dos modelos matemáticos envolvidos mas uma avaliação precisa nas comunicações envolvidas com outros sistemas (Nível 1 PLCs, Nível 2 e Nível 3).

\section{PROJETO PILOTO - SISTEMA DE OTIMIZAÇÃO DOS FORNOS DE REAQUECIMENTO DE PLACAS DO LTQ}

\subsection{Descrições do Sistema do Nível 2 e sua Arquitetura}

O sistema de otimização dos fornos de reaquecimento de placas do Nível 2 opera em regime $24 \times 7$ sendo responsável pelo reaquecimento de todas as placas de forma que seus requisitos de temperatura e uniformidade sejam obtidos para uma laminação estável na produção de bobinas a quente.

A arquitetura de Software e Hardware do projeto piloto consiste em:

- Sistema operacional Open VMS 7.3-2;

- Linguagem de programação: Fontran e C;

- Software básico: Bea Message Queue (Oracle) e Interchange (Rockwell);

- Servidor DS20E 667 Mhz com duas (2) CPUs e 2 GB Ram de memória;

- Storage EVA 4400: Armazenamento externo com acesso aos discos via fibra. A estruturação dos Servidores de Produção e Desenvolvimento/Contingência deste sistema é ilustrada na Figura 4:

* Contribuição técnica ao $18^{\circ}$ Seminário de Automação e TI Industrial, 23 a 26 de setembro de 2014, São Paulo, SP, Brasil. 

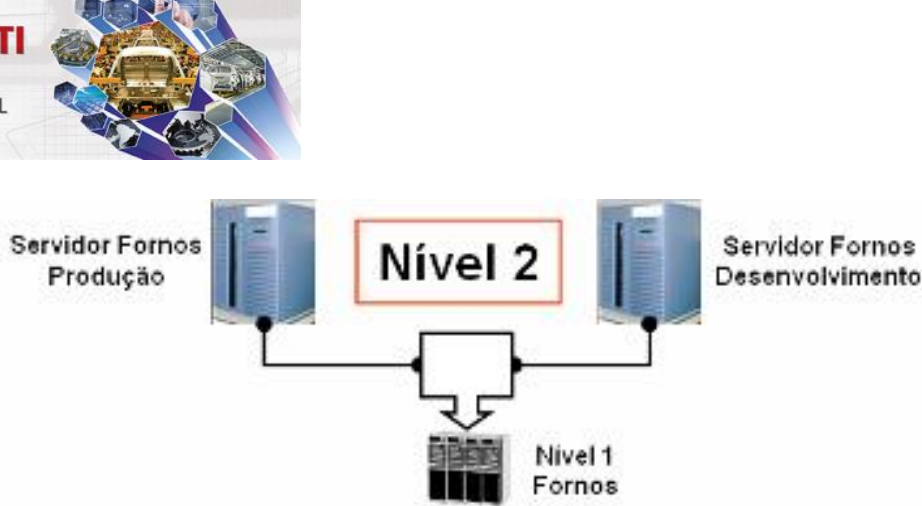

Figura 4 - Arquitetura simplificada do Sistema do Nível 2 dos fornos do LTQ

Esta arquitetura permite a alteração de controle dos fornos do servidor de produção para o servidor de contingência sem perda de dados/rastreamento/controle, ou seja, o servidor de contingência opera totalmente em paralelo, acessando a mesma base de dados do Nível 1, realizando os mesmos cálculos.

Esta característica, aliada ao elevado processamento do sistema (CPU e I/O) foram fundamentais para a caracterização de um piloto que permitisse a validação consistente do produto CHARON.

\subsection{Avaliação Preliminar do Produto CHARON}

A avaliação preliminar do produto foi realizada em 2012 utilizando a infraestrutura existente na companhia, visto não haver recursos suficientes para realizar o teste do produto CHARON nas mesmas condições do servidor Alpha de produção, conforme ilustrado na Tabela 1.

Tabela 1. Infraestrutura da avaliação preliminar

\begin{tabular}{l|l|l}
\hline Descrição & Alpha Server Produção & CHARON (Intel) \\
\hline Processador & $\begin{array}{l}\text { DS20E } \\
\text { 2 CPUs de 666 MHz }\end{array}$ & $\begin{array}{l}\text { Intel Xeon E5630 2.53GHz } \\
\text { (4 núcleos) }\end{array}$ \\
\hline Memória & 2 GB RAM & 6 GB RAM \\
\hline Discos Internos & 36.4 GB- Ultra320 SCSI 15 K & 146 GB - Raid 0 e 1, 15 K \\
\hline $\begin{array}{l}\text { Discos Externos } \\
\text { (Storage) }\end{array}$ & Sim - RAID 5 - Storage HP & Não \\
\hline EVA 4400 & \\
\hline Sistema Operacional & Open VMS 7.3-2 & $\begin{array}{l}\text { Windows 2008 R2 Standard } \\
\text { SP1 }\end{array}$ \\
\hline Comentários Adicionais & & $\begin{array}{l}\text { Versão: 4.1 Build 129-04 } \\
\text { Configuração: } \\
1 \text { núcleo para o Windows } \\
\text { 2 núcleos para o CHARON } \\
\text { 2 núcleos para I/O (disco) }\end{array}$ \\
& & \\
& & \\
\hline
\end{tabular}

A diferença na arquitetura influenciou significativamente na avaliação preliminar, sendo a principal diferença relacionada aos discos, visto que o servidor Alpha Produção opera em uma configuração de alta performance utilizando HBAs (Host Bus Adaptor) que é um adaptador PCl/Fibra ótica que permite acesso aos discos (LUNs) do storage EVA4400 (HP) em uma velocidade 4 Gbps.

Além disto, a configuração dos processadores atendiam somente os requisitos mínimos informados pelo fornecedor, sendo de conhecimento prévio que a performance não seria a mesma do Alpha Server de Produção (DS20E).

* Contribuição técnica ao $18^{\circ}$ Seminário de Automação e TI Industrial, 23 a 26 de setembro de 2014, São Paulo, SP, Brasil. 


\subsection{Avaliação Final do Produto CHARON}

A avaliação final do produto foi realizada no segundo semestre de 2013 utilizando infraestrutura similar ao do servidor Alpha de produção, conforme ilustrado na Tabela 2.

Tabela 2. Infraestrutura da avaliação final

\begin{tabular}{l|l|l}
\hline \multicolumn{1}{c|}{ Descrição } & \multicolumn{1}{c|}{ Alpha Server Produção } & \multicolumn{1}{c}{ CHARON (Intel) } \\
\hline Processador & $\begin{array}{l}\text { DS20E } \\
\text { 2 CPUs de 666 MHz }\end{array}$ & $\begin{array}{l}\text { Dell R610, Intel Xeon X5660 } \\
\text { 2.79 GHz (6 núcleos). }\end{array}$ \\
\hline Memória & 2 GB RAM & 32 GB RAM \\
\hline Discos Internos & 36.4 GB- Ultra320 SCSI 15 K & 200 GB - Raid 0 e 1, 15K \\
\hline $\begin{array}{l}\text { Discos Externos } \\
\text { (Storage) }\end{array}$ & Sim - RAID 5 - Storage HP & $\begin{array}{l}\text { Sim - RAID 5 - Storage HP } \\
\text { EVA 4400 }\end{array}$ \\
\hline SVA 4400 & $\begin{array}{l}\text { Windows 2008 R2 Standard } \\
\text { SP1 }\end{array}$ \\
\hline Comentários Adicionais & Open VMS 7.3-2 & $\begin{array}{l}\text { Versão: 4.2 Build 141-03. } \\
\text { Configuração: } \\
\text { 1 núcleo para o Windows } \\
\text { 2 núcleos para o CHARON } \\
\text { 2 núcleos para I/O (disco) }\end{array}$ \\
& & \\
& & \\
& & \\
\hline
\end{tabular}

\section{RESULTADOS E DISCUSSÃO}

\subsection{Resultados da Avaliação Preliminar do Produto CHARON}

Como esperado e informado pelo fornecedor do produto, o desempenho não foi o mesma do servidor Alpha de Produção (DS20E).

A lentidão no processamento era visível nas telas operacionais do Sistema no qual um evento era atualizado na tela somente após 10 segundos. Este atraso foi considerado elevado, conforme ilustrado na tela de rastreamento físico (Figura 5) na qual se observa a ausência de uma placa física na sequência de enfornamento.

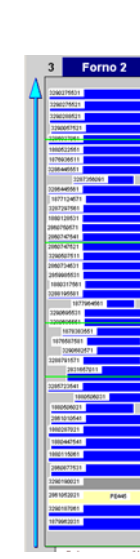

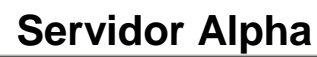

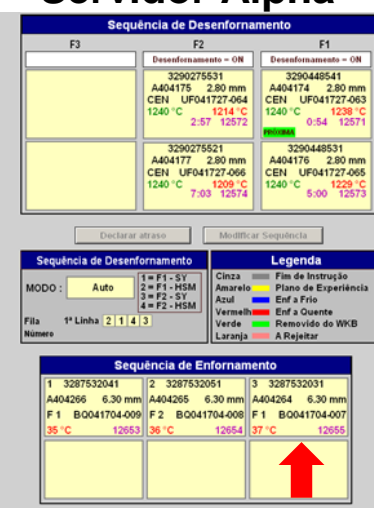

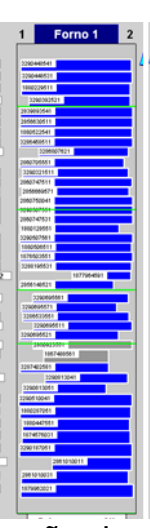

Servidor CHARON

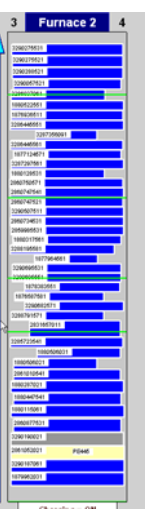

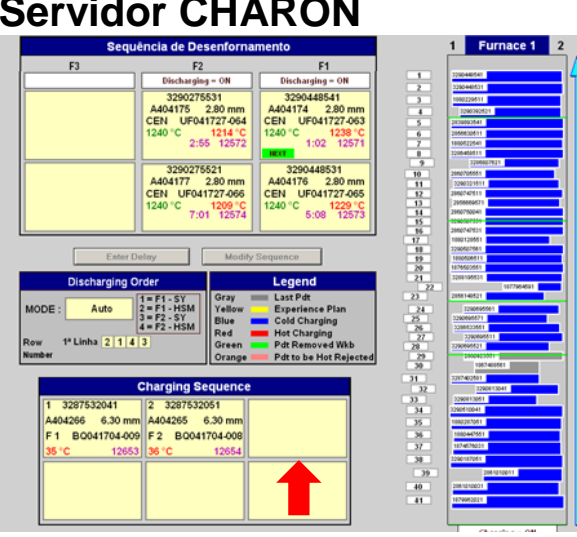

Figura 5 - Atraso na atualização da tela de rastreamento físico das placas

Além disso, o monitoramento dos processos em execução no Servidor (Open VMS) do CHARON mostrou acúmulo de eventos não processados, conforme ilustrado na

\footnotetext{
* Contribuição técnica ao $18^{\circ}$ Seminário de Automação e TI Industrial, 23 a 26 de setembro de 2014, São Paulo, SP, Brasil.
} 
Figura 6, o que impossibilita a visualização nas telas que permitem a seleção de algum dado específico desejado pelo usuário. A Tabela 3 ilustra o comparativo dos tempos de compilação e link, porém este não é um item impeditivo de avaliação de desempenho (Somente referência).

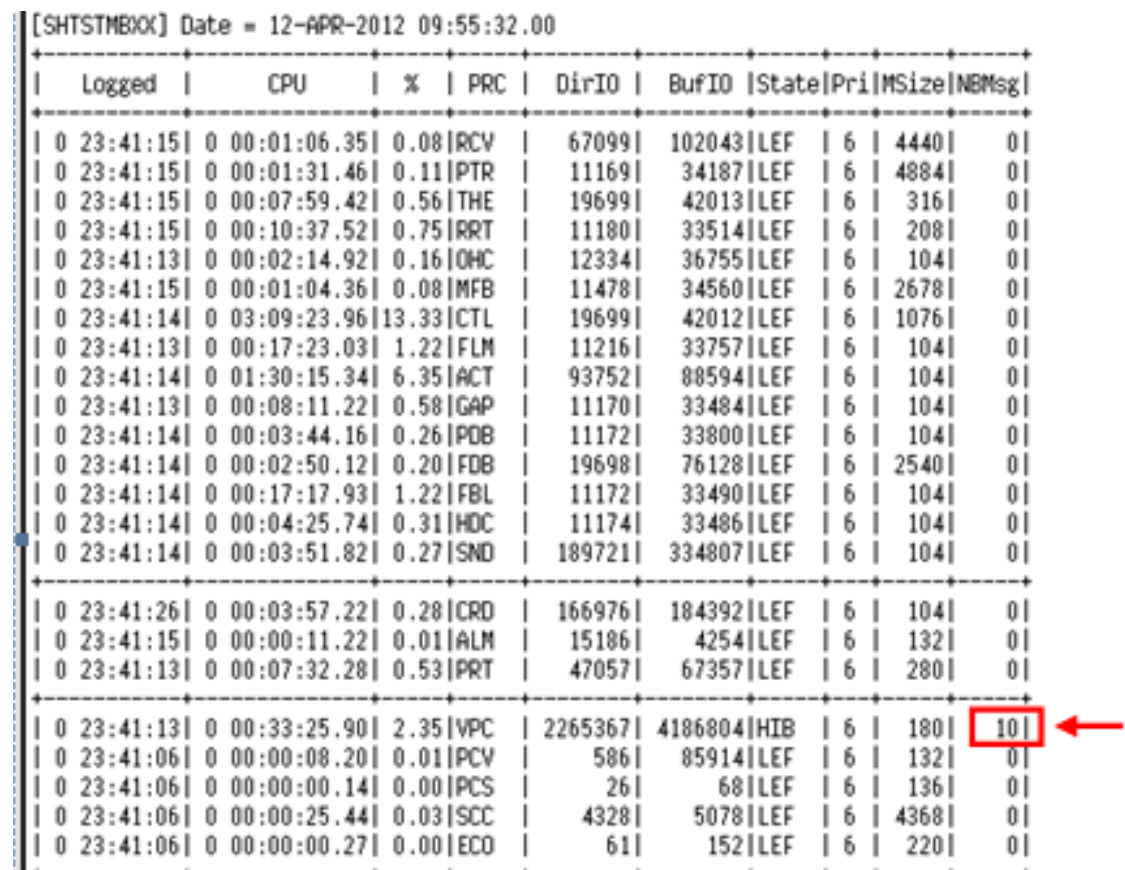

Figura 6 - Mensagens pendentes não processadas

Tabela 3. Tempo em segundos na compilação e link do sistema

\begin{tabular}{|c|c|c|c|c|c|c|c|c|c|c|c|c|}
\hline \multicolumn{13}{|c|}{ COMPARATIVO CHARON x HSMDV2 } \\
\hline Descrição & \begin{tabular}{|l|} 
Plataforma \\
\end{tabular} & Med 1 & Med 2 & Med 3 & Med 4 & Med 5 & Med 6 & Média & \begin{tabular}{|l} 
Desvio Padrão \\
\end{tabular} & Amplitude & Min & Máx \\
\hline \multirow{2}{*}{$\begin{array}{l}\text { Compilação Total de } \\
\text { um processo. }\end{array}$} & CHARON & 25 & 33 & 25 & 29 & 36 & 33 & 30 & 4.58 & 11 & 25 & 36 \\
\hline & Alpha Server & 18 & 11 & 14 & 10 & 11 & 10 & 12 & 3.14 & 08 & 10 & 18 \\
\hline \multirow{2}{*}{$\begin{array}{l}\text { Link de um } \\
\text { processo }\end{array}$} & CHARON & 01 & 01 & 00 & 00 & 02 & 01 & 0.83 & 0.75 & 02 & 00 & 02 \\
\hline & Alpha Server & 01 & 01 & 00 & 01 & 01 & 01 & 0.83 & 0.41 & 01 & 00 & 01 \\
\hline \multirow{2}{*}{$\begin{array}{l}\text { Compilação Total de } \\
\text { todo aplicativo. }\end{array}$} & CHARON & 652 & 615 & 683 & 692 & 724 & 786 & 692 & 59.11 & 171 & 615 & 786 \\
\hline & Alpha Server & 304 & 297 & 292 & 301 & 301 & 308 & 301 & 5.54 & 16 & 292 & 308 \\
\hline
\end{tabular}

Conforme esperado, no quesito desempenho o produto não conseguiu atingir o mesmo nível do ambiente real, fortemente influenciado pela infraestrutura utilizada. Trata-se de um produto com potencial para atingir ao objetivo proposto: ser uma alternativa viável para futuras substituições de hardware ALPHA.

A evolução do projeto foi realizada em infraestrutura similar ao ambiente de produção do mesmo sistema conforme descrito na sessão subsequente deste trabalho.

\subsection{Resultados da Avaliação Final do Produto CHARON}

Em condições de infraestrutura similar, o desempenho do produto CHARON atingiu resultados bem mais expressivos quando comparado com os testes preliminares. Foram realizadas as seguintes avaliações de desempenho:

\footnotetext{
* Contribuição técnica ao $18^{\circ}$ Seminário de Automação e TI Industrial, 23 a 26 de setembro de 2014,
} São Paulo, SP, Brasil. 


\subsubsection{Tempo para o Cálculo do Valor de PI}

Esta avaliação foi realizada durante uma parada programada sem nenhuma carga em ambos os sistemas. O tempo obtido em segundos refere-se ao cálculo do valor de PI com 40000 casas decimais.

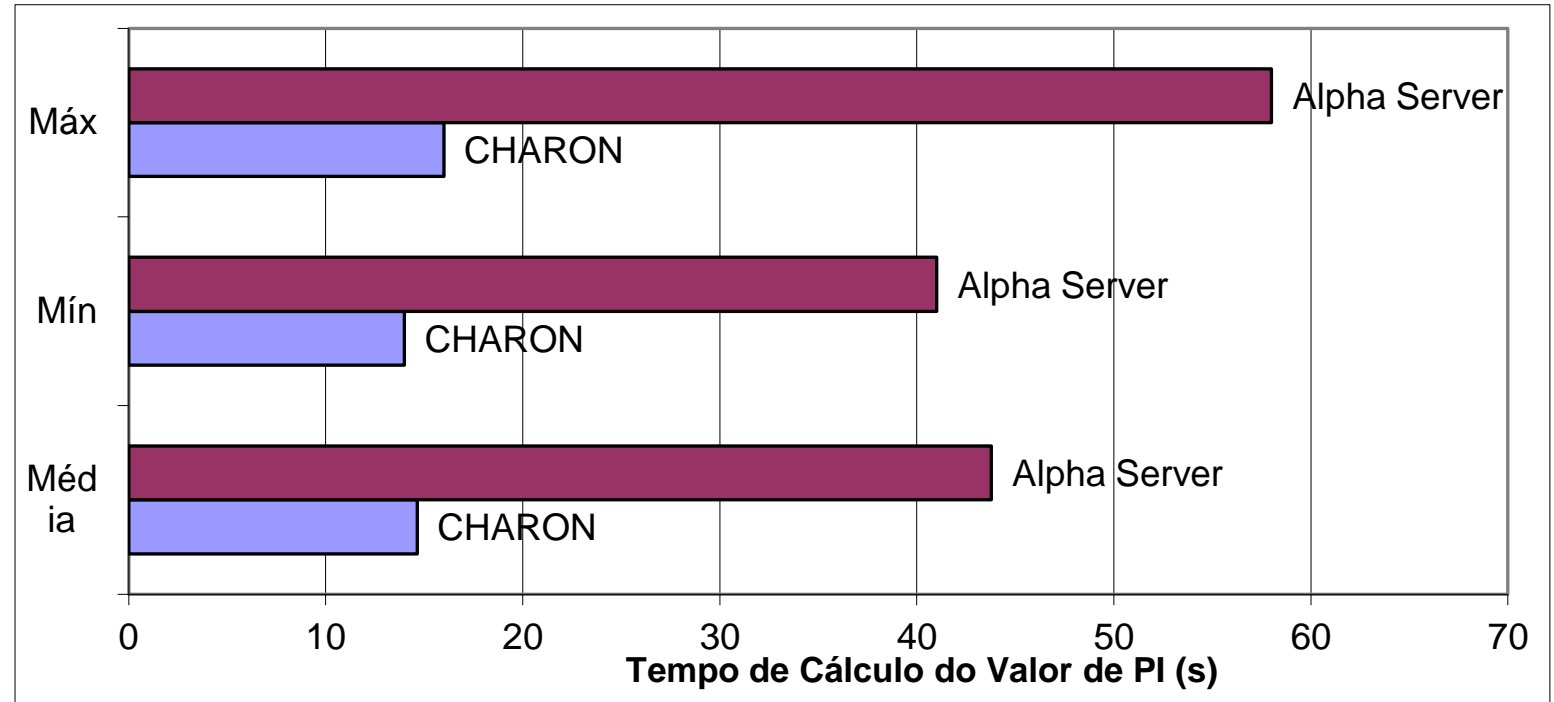

Figura 7 - Tempo para calcular o valor de PI

Observa-se na Figura 7 que o desempenho do CHARON (Média $15 \mathrm{~s}$ ) foi melhor quando comparado ao servidor Alpha original (Média $44 \mathrm{~s}$ ).

\subsubsection{Tempo de Compilação e Link do Sistema}

Esta avaliação foi realizada durante laminação somente para efeito comparativo coma avaliação preliminar do produto e demonstrada na Figura 8.

Nesta nova análise, o produto CHARON apresentou um desempenho inferior (Média 362 s) em relação ao servidor Alpha (Média 270 s), porém em relação à avaliação preliminar o CHARON melhorou significativamente em desempenho, reduzindo o tempo médio de $692 \mathrm{~s}$ para $362 \mathrm{~s}$.

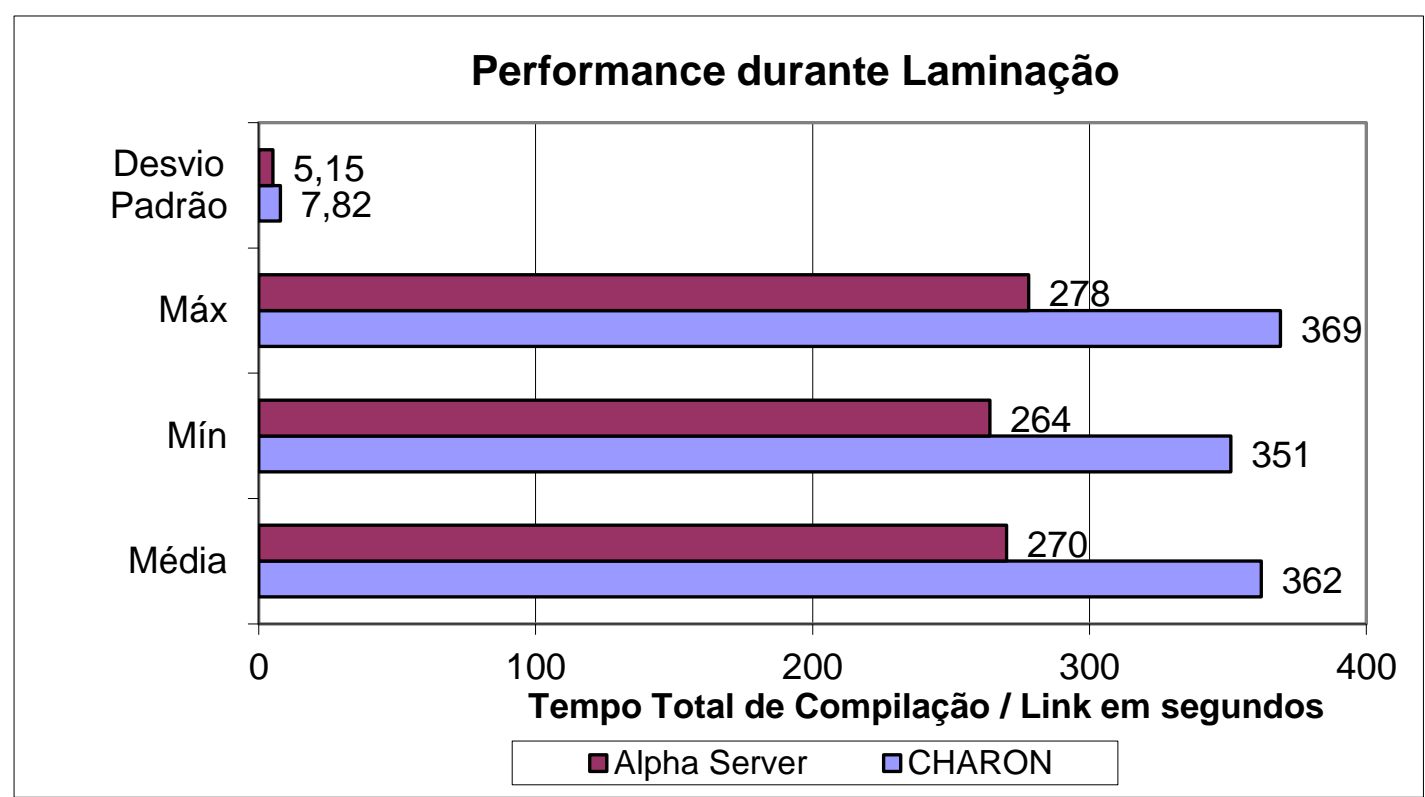

Figura 8 - Tempo total de Compilação e Link do aplicativo em segundos

* Contribuição técnica ao $18^{\circ}$ Seminário de Automação e TI Industrial, 23 a 26 de setembro de 2014, São Paulo, SP, Brasil. 


\subsubsection{Acúmulo de eventos não processados}

Esta avaliação é essencial na análise de desempenho do produto. Durante alguns meses, avaliou-se o CHARON em paralelo com o servidor Alpha Server e não foram detectados atrasos de atualização em telas e nem acúmulo de eventos não processados.

Uma vez que o produto foi aprovado neste quesito de desempenho, estabeleceu-se uma forma para avaliação do desempenho do aplicativo de otimização do Nível 2 de forma quantitativa conforme descrito na sessão a seguir.

\subsubsection{Tempo de Processamento de Eventos}

Esta avaliação foi definida com o objetivo de quantificar o tempo médio de processamento dos eventos de entrada e sua conclusão antes de liberar o sistema para avaliação no ambiente produtivo.

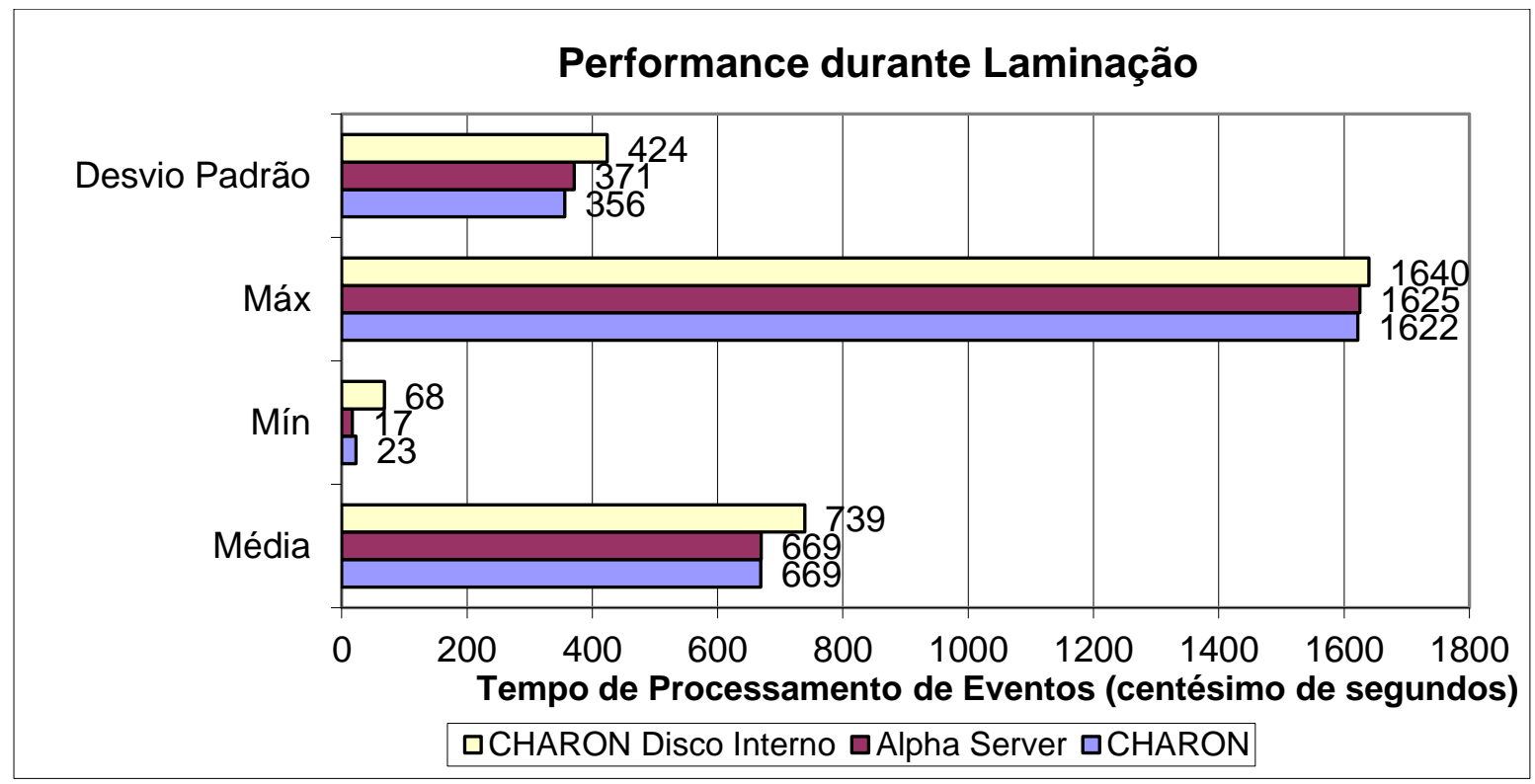

Figura 9 - Tempo de Processamento dos eventos em centésimo de segundos

Observa-se no gráfico da Figura 9 que o tempo médio de processamento dos eventos com o CHARON e o servidor Alpha operando através de discos localizados no Storage EVA 4400 foram iguais (669 cs).

A avaliação também foi realizada para comparar o tempo médio de processamento dos eventos operando o CHARON em modo de contingência, ou seja, em funcionamento pelos discos internos do próprio servidor Intel. O desempenho em relação ao storage é inferior, porém o sistema se manteve operacional com certa lentidão esperada.

\section{CONCLUSÃO}

Primeiramente, para avaliar este produto, a infraestrutura deve ser o mais próximo do ambiente real em uso na operação e controle dos equipamentos. A avaliação em aspectos inferiores somente deve ser utilizada para adquirir experiência no produto. $\mathrm{Na}$ avaliação final da solução, podemos destacar os seguintes quesitos:

- Compatibilidade e portabilidade: o produto foi completamente aderente. Não se detectou problemas nos aplicativos e modelos matemáticos utilizados na otimização do reaquecimento de placas.

* Contribuição técnica ao $18^{\circ}$ Seminário de Automação e TI Industrial, 23 a 26 de setembro de 2014, São Paulo, SP, Brasil. 
- Robustez/estabilidade: o produto se manteve em produção no controle dos fornos de reaquecimento de placas do LTQ por aproximadamente dois (2) meses atendendo as requisitos operacionais e de otimização.

O produto apresentou uma falha no driver da sua chave de proteção (hardlock USB) provocando um desligamento inesperado do emulador. A falha foi rapidamente corrigida por aplicação de patches, porém foi solicitada ao fabricante do produto a avaliação de uma solução mais robusta (ou a eliminação deste tipo de proteção) para se evitar impactos operacionais.

O produto CHARON mostrou-se bem aderente, no qual a recuperação dos backups, execução das aplicações, interfaces de comunicação e outros recursos foram emulados de forma transparente, com pouco esforço de configuração.

A expansão da validação do produto CHARON já está planejada para 2014, envolvendo as demais áreas produtivas da empresa (Redução, Utilidades e Aciaria) assim como a validação do mesmo no LTQ no Sistema de Nível 2 do desenvolvimento do Controle de Perfil, Coroa e Planicidade das bobinas.

\section{REFERÊNCIAS}

1 Stromasys Virtualization Technologies. Disponível em <http://www.stromasys.com/> Acesso em 10/06/2014

* Contribuição técnica ao $18^{\circ}$ Seminário de Automação e TI Industrial, 23 a 26 de setembro de 2014, São Paulo, SP, Brasil. 